\title{
Spatial Patterns and Bifurcation Analysis of a Diffusive Tumour-immune Model
}

\author{
Jingjing Wang, Hongchan Zheng*, Yunfeng Jia and Hong-Kun Xu
}

\begin{abstract}
In this paper, a diffusive tumour-immune model is presented. By comparing the effect of Neumann boundary conditions and Dirichlet boundary conditions on the stability of trivial equilibrium, we derive that the former can provide more mechanisms for spatial pattern formation of the model. By taking the diffusion rate of tumour cells as a parameter, we first give the local and global steady-state bifurcations emitting from the positive equilibrium of the model. Then the stability of the bifurcation solution is discussed by computing the second derivative of an appropriate function, which is different from the general case. Furthermore, numerical simulations provide an indication of the wealth of patterns that the system can exhibit. In particular, periodic oscillation and spot-like patterns can be observed in one-dimensional and two-dimensional simulations, respectively. All results obtained reveal the mechanism of interaction between tumour cells and immune system, which have profound significance for the development of tumour immunotherapy.
\end{abstract}

\section{Introduction}

Malignant tumours of cancer are caused by abnormal cell proliferation. In the past decades, as the number of cancer patients has increased, researchers have been focusing on cancer treatment in experimental and theoretical medicine. Specially, understanding the dynamics of the interaction between tumour cells and immune cells is one of the most fundamental issues in cancer treatment 25]. However, the interaction behaviour between tumour cells and immune cells is very complex in clinical trials since the cells may not behave as predicted in vitro trials. Fortunately, mathematical modeling has been shown to contribute to a more realistic understanding of the properties of such complex biological systems [1]. Therefore, a large number of tumour-immune systems have been established to understand tumourigenesis and tumour progression in recent years $2,4,23,34$.

In [20], Kuznetsov et al. proposed a system of ordinary differential equations (ODEs) tumour model including tumour cells and immune system cells (commonly called effector

Received April 13, 2020; Accepted September 21, 2020.

Communicated by Cheng-Hsiung Hsu.

2010 Mathematics Subject Classification. 37M20, 58J55, 35K57.

Key words and phrases. tumour-immune model, patterns, bifurcation, stability, numerical simulations. The work is supported in part by the National Natural Science Foundations of China (11771262, 11671243). *Corresponding author. 
cells), they studied the dynamics and the phenomena of oscillation and dormancy of immunogenic tumours. The model is described by

$$
\left\{\begin{array}{l}
\frac{d x}{d t}=r x(1-\eta x)-a x y \\
\frac{d y}{d t}=\frac{b x y}{1+\omega x}-c x y-d y
\end{array}\right.
$$

where $x$ and $y$ are concentrations of tumour cells and effector cells, respectively, $r$ is the intrinsic growth rate of tumour cells, $1 / \eta$ is the carrying capacity of tumour cells, $a$ is the rate at which the effector cells bind to the tumour cells, $b$ denotes the maximum immune response rate due to the presence of tumour cells, $\omega$ is the steepness of immune response, $c$ is the inactivation rate of effector cells, and $d$ is the death rate of effector cells. Here all the parameters are positive.

In order to reduce the number of parameters, we rescale 1.1). Set

$$
t=\frac{1}{r} \widetilde{t}, \quad x=\frac{u}{\eta}, \quad y=\frac{r}{a} v, \quad e=\frac{d}{r}, \quad s=\frac{c}{\eta r}, \quad q=\frac{b}{r \omega}, \quad p=\frac{\eta}{w},
$$

and still denote $\widetilde{t}$ by $t$, then the model $(1.1)$ becomes into the following form

$$
\left\{\begin{array}{l}
\frac{d u}{d t}=u(1-u)-u v, \\
\frac{d v}{d t}=\frac{q u v}{p+u}-s u v-e v,
\end{array}\right.
$$

where $u$ and $v$ represent the concentrations of tumour cells and effector cells, respectively. $q, p, s, e$ can be interpreted as normalized maximum immune response rate, steepness of immune response, inactivation rate of effector cells and death rate of effector cells, respectively. Based on model (1.2), Yang et al. 36 considered the influence of noise on tumour immunodynamics by using a stochastic differential equation. They obtained that the combinations of immunotherapy and chemotherapy or noise can dominate the evolution of tumours by investigating tumor free solution and global positive solution of the system. Starkov and Krishchenko 28 studied the impact of healthy host cells on the tumour growth. By calculating the upper and lower bounds for the immune cells population and the sufficient conditions for trajectories from the positive domain of feasible initial conditions to the equilibrium points, the effects of healthy host cells on the tumour cells growth and immunotherapy were explored.

Although model (1.2) and its extended models in 28,36 have been studied well, these models are all ODE models, and they have a limitation: they are all assumed as a priori and lack the basis of the internal mechanism of tumour growth. For instance, the volume of tumour cells cannot increase indefinitely during its growth, which is caused by the balance of cell proliferation and death in tumour, but the ODE models cannot reflect this feature. With that in mind, by considering the reaction and diffusion of nutrients and inhibitors, 
and the reproduction and death of cells caused by them, a system of partial differential equations (PDEs) was proposed by Greenspan [13] to describe the tumour growth. He proved that the PDE system is not only better at describing tumour growth, but also better at responding to the inhibition caused by the mutual movement of different cell groups. So the tumour model of PDE system is more practical, which mainly consists of two formulae, the first simulates the diffusion and proliferation of tumour cells while the second is used as the boundary condition. For boundary conditions, many people have given different types of boundaries under different biological backgrounds, such as the homogeneous Dirichlet boundary representing that the cell concentration on the boundary of the model region can be ignored, the homogeneous Neumann boundary referring that there is no-flux of cell on the boundary, and the free boundary indicating that the size and shape of the tumour do not change with time in the dormant state, while the cells in the tumour are alive and undergo the process of proliferation and movement before death, etc. Recently, many descriptions of tumour growth have been presented by using PDE systems with different boundary conditions. For example, Su et al. 29] gave some tumour models with homogeneous Dirichlet boundary conditions. Through numerical simulation, the abundant dynamic behaviors of the model are revealed. Jiang et al. 18 studied a Cahn-Hilliard-Darcy system with homogeneous Neumann boundary conditions and non-autonomous mass source term that models tumour growth. They proved the existence and qualitative behavior of solutions. Friedman and Lam 10 presented a freeboundary tumour model with angiogenesis. They discussed the existence and uniqueness of stationary solution, and proved the global asymptotic stability of steady states. For more tumour-immune models of PDE systems, one may further refer to [3, 7, 11, 35], etc.

Motivated by the works mentioned above, in this paper, we add the diffusion terms $\Delta u$ and $\Delta v$ into system $(1.2)$. Further, when tumour cells interact with effector cells, they undergo maturation and migrate from their initial position to the model boundary. This causes the concentrations of cells on the boundary are very small. Hence, the distribution of cells on the boundary can be ignored. This implies that we can impose the homogeneous Dirichlet boundary conditions $u=v=0$ on $(1.2)$, then we can get the following problem

$$
\begin{cases}\frac{\partial u}{\partial t}=\sigma_{1} \Delta u+u(1-u)-u v, & x \in \Omega, t>0, \\ \frac{\partial v}{\partial t}=\sigma_{2} \Delta v+\frac{q u v}{p+u}-s u v-e v, & x \in \Omega, t>0, \\ u(x, t)=v(x, t)=0, & x \in \partial \Omega, t>0, \\ u(x, 0)=u_{0}(x) \geq, \not \equiv 0, \quad v(x, 0)=v_{0}(x) \geq, \not \equiv 0, & x \in \Omega,\end{cases}
$$

where $\Omega$ is a bounded domain in $\mathbb{R}^{n}$ with smooth boundary $\partial \Omega, \sigma_{1}$ and $\sigma_{2}$ are the diffusion rates corresponding to $u$ and $v$, respectively, and other parameters are the same as in system $(1.2)$. The admissible initial data $u_{0}(x)$ and $v_{0}(x)$ are continuous functions in $\bar{\Omega}$. 
On the other hand, in reality, the positive thing we expect is that the tumour cells do not spread to other tissues. Hence, to prevent tumour cells from spreading out of the model boundary and to keep the boundary conditions of tumour cells and effector cells consistent, the homogeneous Neumann boundary conditions $\frac{\partial u}{\partial \nu}=\frac{\partial v}{\partial \nu}=0$ are imposed on (1.2). And thus (1.2) becomes

$$
\begin{cases}\frac{\partial u}{\partial t}=\sigma_{1} \Delta u+u(1-u)-u v, & x \in \Omega, t>0 \\ \frac{\partial v}{\partial t}=\sigma_{2} \Delta v+\frac{q u v}{p+u}-s u v-e v, & x \in \Omega, t>0 \\ \frac{\partial u}{\partial \nu}=\frac{\partial v}{\partial \nu}=0, & x \in \partial \Omega, t>0 \\ u(x, 0)=u_{0}(x) \geq, \not \equiv 0, \quad v(x, 0)=v_{0}(x) \geq, \not \equiv 0, & x \in \Omega .\end{cases}
$$

Here $\nu$ is the outward unit normal vector on $\partial \Omega$, and other parameters are the same as in system 1.3 . For more detailed biological motivations and meanings for Dirichlet boundary conditions and Neumann boundary conditions, one can see $3,5,8,9,11,18,22$, $29,30,32$, etc.

Pattern formation is a very interesting nonlinear phenomenon, it can be used to describe the structure changes of interacting species in ecology and chemical reactants. For instance, in tumour-immune models, pattern formation can be used to judge the proliferation and death of tumour cells over time. For the diffusive biological systems, the Turing instability and bifurcation phenomena are two well-known mechanisms of pattern formation. So they have been paid much attention in recent decades, see 16, 19, 31 for examples.

As is known to all, boundary conditions can affect the stability of trivial equilibrium and thus affect pattern formation of the system. However, we are interested in which of the two systems (1.3) and (1.4) with different boundaries, will generate more mechanisms for spatial pattern formation at the trivial equilibrium. Therefore, we consider the effect of boundary conditions on the stability of trivial equilibrium to systems (1.3) and (1.4) in this study. On the other hand, steady-state bifurcation can also generate rich spatial patterns and dynamical behaviors, hence it has been considered by many authors. For example, some authors studied the steady-state bifurcation emitting from simple eigenvalue [6, 15, 17], and some authors investigated the steady-state bifurcation emitting from double eigenvalues $14,21,33$. In this paper, our main contribution is a detailed bifurcation analysis emitting from simple eigenvalue for steady-state system of (1.4) by using the approach in [6, 15, 17] since the eigenvalue of a certain operator is only simple under the condition that the biological meaning is satisfied. Moreover, since the stability of the steady-states can determine the progress of population evolution in a large extent, we also discuss the stability of bifurcating solution. Different from the conventional approach introduced in [27], we compute the second derivative of the appropriate function in an- 
alyzing the stability since it is not enough to only compute the first derivative for our model, a detailed explanation will be given in Section 4 .

This paper is organized as follows. In Section 2, we study the effect of boundary conditions on the stability of equilibria to systems (1.3) and (1.4). The long time behavior of solutions to system (1.4) is given in Section 3. In Section 4, we investigate the local and global bifurcation of steady-state system of (1.4) and the stability of bifurcation solution. Some numerical examples and patterns are presented in Section 5 . Section 6 gives the conclusion and future work to end the investigation.

\section{Effect of boundary conditions on the stability of equilibria}

In this section, we analyze the stability of equilibria for systems $(1.3)$ and $(1.4)$ by using the stability theory of eigenvalues [12]. Firstly, we discuss the stability of trivial equilibrium.

Clearly, systems (1.3) and (1.4) have trivial equilibrium $U_{0}=(0,0)$. Linearizing the system 1.3 at $U_{0}$ gives

$$
\left(\begin{array}{cc}
\frac{\partial}{\partial t} & 0 \\
0 & \frac{\partial}{\partial t}
\end{array}\right)\left(\begin{array}{l}
u \\
v
\end{array}\right)-L_{0}\left(\begin{array}{l}
u \\
v
\end{array}\right)=0 \quad \text { with } \quad L_{0}=\left(\begin{array}{cc}
\sigma_{1} \Delta+1 & 0 \\
0 & \sigma_{2} \Delta-e
\end{array}\right) .
$$

Suppose that $0<\mu_{0} \leq \cdots \leq \mu_{j} \leq \cdots$ are the sequence of eigenvalues of $-\Delta$ with homogeneous Dirichlet boundary conditions on $\Omega$, where each $\mu_{j}$ has multiplicity $n_{j} \geq 1$. Let $\varphi_{j k}, 1 \leq k \leq n_{j}$ be the normalized eigenfunctions corresponding to $\mu_{j}$. Then the set $\left\{\varphi_{j k}\right\}, j \geq 0,1 \leq k \leq n_{j}$ forms a complete orthonormal basis in $L_{2}(\Omega)$. Assume that $\left(\Psi_{1}(x), \Psi_{2}(x)\right)$ is an eigenfunction of $L_{0}$ corresponding to an eigenvalue $\alpha$. Then $L_{0}\left(\Psi_{1}(x), \Psi_{2}(x)\right)^{T}=\alpha\left(\Psi_{1}(x), \Psi_{2}(x)\right)^{T}$. Set

$$
\Psi_{1}(x)=\sum_{0 \leq j \leq \infty}^{1 \leq k \leq n_{j}} a_{j k} \varphi_{j k}, \quad \Psi_{2}(x)=\sum_{0 \leq j \leq \infty}^{1 \leq k \leq n_{j}} b_{j k} \varphi_{j k}
$$

We have

$$
\sum_{0 \leq j \leq \infty}^{1 \leq k \leq n_{j}}\left(\begin{array}{cc}
-\sigma_{1} \mu_{j}+1-\alpha & 0 \\
0 & -\sigma_{2} \mu_{j}-e-\alpha
\end{array}\right)\left(\begin{array}{c}
a_{j k} \\
b_{j k}
\end{array}\right) \varphi_{j k}=\sum_{0 \leq j \leq \infty}^{1 \leq k \leq n_{j}} \mathbf{P}\left(\begin{array}{l}
a_{j k} \\
b_{j k}
\end{array}\right) \varphi_{j k}=0 .
$$

It is easy to see that $\alpha$ is an eigenvalue of $L_{0}$ if and only if the determinant of the matrix $\mathbf{P}$ is zero for some $j \geq 0$, that is,

$$
\alpha^{2}-M_{1} \alpha+M_{2}=0,
$$

where

$$
\begin{aligned}
& M_{1}=-\mu_{j}\left(\sigma_{1}+\sigma_{2}\right)+1-e, \\
& M_{2}=\sigma_{1} \sigma_{2} \mu_{j}^{2}+\left(e \sigma_{1}-\sigma_{2}\right) \mu_{j}-e .
\end{aligned}
$$


Now, we discuss the stability of trivial equilibrium $U_{0}=(0,0)$ for systems $(1.3)$ and (1.4), which is described as the following Theorem 2.1.

Theorem 2.1. (1) The equilibrium $U_{0}$ of system (1.3) is locally asymptotically stable if $\sigma_{1}>1 / \mu_{0}$ and unstable if $\sigma_{1}<1 / \mu_{0}$.

(2) The equilibrium $U_{0}$ of system (1.4) is unstable.

Proof. Let $\mu_{ \pm}$be two roots of $(2.3)$. Then $\mu_{-}=\frac{-e}{\sigma_{2}}$ and $\mu_{+}=\frac{1}{\sigma_{1}}$. It is clear that $\frac{1}{\sigma_{1}}>\frac{1-e}{\sigma_{1}+\sigma_{2}}$. Since $\mu_{j}$ is monotonically increasing with respect to $j$, if $\mu_{0}>\mu_{+}$, then from (2.2) and (2.3) we have $M_{1}<0$ and $M_{2}>0$ for $j \geq 0$. This means that the equilibrium $U_{0}$ of system $(1.3)$ is locally asymptotically stable when $\sigma_{1}>1 / \mu_{0}$. And clearly, $U_{0}$ is unstable if $\sigma_{1}<1 / \mu_{0}$. This completes the proof of (1).

Moreover, for system (1.4), we can also get (2.1) but for $\mu_{0}=0$, and then we can easily obtain (2) holds.

Remark 2.2. Theorem 2.1 presents the effect of Dirichlet boundary conditions and Neummann boundary conditions on the stability of trivial equilibrium $U_{0}$ of systems $(1.3)$ and (1.4). Particularly, we see from Theorem 2.1(1) that the equilibrium $U_{0}$ of the system (1.3) is stable when the diffusion rate $\sigma_{1}$ of tumour cells is large and unstable when $\sigma_{1}$ is small. However, according to Theorem 2.1 (2), we know that the equilibrium $U_{0}$ of the system (1.4) is always unstable. These indicate that the Dirichlet boundary conditions have an important effect on the stability of equilibrium $U_{0}$ and the Neumann boundary conditions cannot affect the instability of equilibrium $U_{0}$. On the other hand, Theorem 2.1 (1) shows a hyperbolic curve $\sigma_{1}=1 / \mu_{0}$ in the first quadrant on the $\sigma_{1}-\mu_{0}$ parameter plane, and clearly the curve separates the stable and unstable regions of the equilibrium $U_{0}$ of system (1.3), see Figure 2.1. Moreover, from Theorem 2.1(2) we know that the equilibrium $U_{0}$ of the system (1.4) is always unstable in the first quadrant on the $\sigma_{1}-\mu_{0}$ parameter plane.

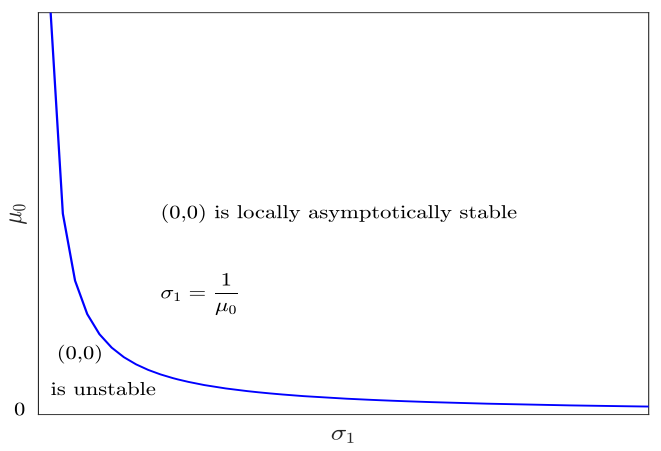

Figure 2.1: The stable/unstable region of equilibrium $U_{0}=(0,0)$ for system 1.3 in $\sigma_{1}-\mu_{0}$ parameter plane. 
Remark 2.3. Biologically, the instability of trivial equilibrium $U_{0}=(0,0)$ has two manifestations in clinical trials: (i) If there are no effector cells, then the tumour cells will not die out once they invade; (ii) The tumour cells and effector cells may coexist, and the presence of tumour cells cannot pose a threat to human health. These manifestations not only provide some mechanisms for spatial pattern formation, but also have great significance to the development of tumour immunotherapy in clinical trials. Thus, Neumann boundary conditions are more preferable for the survival of cell population than the Dirichlet boundary conditions in this diffusive tumour-immune system. Next, we study the pattern formation of the system (1.4). To this end, we first investigate the existence and stability of semi-trivial and positive equilibria for system (1.4).

Lemma 2.4. (1) System (1.4) always has semi-trivial equilibrium $U_{1}^{*}=\left(u_{1}^{*}, v_{1}^{*}\right)=(1,0)$;

(2) If $q=(\sqrt{s p}+\sqrt{e})^{2}$ and $s>$ pe, then system (1.4) has a unique positive equilibrium $U_{2}^{*}=\left(u_{2}^{*}, v_{2}^{*}\right)=\left(\sqrt{\frac{p e}{s}}, 1-\sqrt{\frac{p e}{s}}\right)$;

(3) If $(\sqrt{s p}+\sqrt{e})^{2}<q<\min \{(s+e)(1+p), s(2+p)+e\}$, then system (1.4) has two positive equilibria $U_{3}^{*}=\left(u_{3}^{*}, v_{3}^{*}\right)=\left(u_{3}^{*}, 1-u_{3}^{*}\right)$ and $U_{4}^{*}=\left(u_{4}^{*}, v_{4}^{*}\right)=\left(u_{4}^{*}, 1-u_{4}^{*}\right)$, where $u_{3}^{*}=\frac{q-s p-e+\sqrt{(q-s p-e)^{2}-4 s p e}}{2 s}, u_{4}^{*}=\frac{q-s p-e-\sqrt{(q-s p-e)^{2}-4 s p e}}{2 s}$;

(4) If $(s+e)(1+p)<q<s(2+p)+e$, then system (1.4) has a unique positive equilibrium $U_{4}^{*}=\left(u_{4}^{*}, v_{4}^{*}\right)=\left(u_{4}^{*}, 1-u_{4}^{*}\right)$.

Denote $U=(u, v)^{T}$ and

$$
H(U)=\left(\begin{array}{c}
\sigma_{1} \Delta u+u(1-u)-u v \\
\sigma_{2} \Delta v+\frac{q u v}{p+u}-s u v-e v
\end{array}\right)
$$

The linearized operator of $H(U)$ at $U_{i}^{*}=\left(u_{i}^{*}, v_{i}^{*}\right), i=1,2,3,4$ are

$$
H_{U}\left(U_{i}^{*}\right)=\left(\begin{array}{cc}
\sigma_{1} \Delta+1-2 u_{i}^{*}-v_{i}^{*} & -u_{i}^{*} \\
\mathfrak{f}_{i} & \sigma_{2} \Delta+\frac{q u_{i}^{*}}{p+u_{i}^{*}}-s u_{i}^{*}-e
\end{array}\right)
$$

with

$$
\mathfrak{f}_{i}=v_{i}^{*} \frac{q p-s\left(p+u_{i}^{*}\right)^{2}}{\left(p+u_{i}^{*}\right)^{2}}, \quad i=1,2,3,4 .
$$

It is clear that $\mathfrak{f}_{1}=\mathfrak{f}_{2}=0$. Let $0=\mu_{0}<\mu_{1} \leq \mu_{2} \leq \cdots \leq \mu_{j} \leq \cdots$ be the eigenvalues of $-\Delta$ with homogeneous Neumann boundary conditions on $\Omega$. Assume that $\mathfrak{f}_{i}<0$ and $\mu_{1}<-\mathfrak{f}_{i} / \sigma_{2}, i=3,4$. Then there exists a largest positive integer $j_{\tau}^{(i)}$ such that $\mu_{j}<-\mathfrak{f}_{i} / \sigma_{2}$ for $1 \leq j \leq j_{\tau}^{(i)}$ and $i=3$, 4. Let

$$
\delta_{j}^{(i)}=\frac{-u_{i}^{*} \sigma_{2} \mu_{j}-\mathfrak{f}_{i} u_{i}^{*}}{\sigma_{2} \mu_{j}^{2}}, \quad \bar{\delta}^{(i)}=\min _{1 \leq j \leq j_{\tau}^{(i)}} \delta_{j}^{(i)}, \quad i=3,4 .
$$


Next we introduce the local stability of equilibria $U_{i}^{*}=\left(u_{i}^{*}, v_{i}^{*}\right), i=1,2,3,4$, see Theorem 2.5. It is worth pointing out that the stability theory of eigenvalues is the main argument for the Theorem 2.5, so the eigenvalues $\mu_{j}, j \geq 0$ must be applied in the proof. However, to reflect the effect of diffusion, we only consider the case of $\mu_{j}, j \geq 1$.

Theorem 2.5. (1) If $q<(s+e)(p+1)$, then the equilibrium $U_{1}^{*}$ of system (1.4) is asymptotically stable;

(2) Let the conditions of Lemma 2.4(2) hold. Then the equilibrium $U_{2}^{*}$ of system (1.4) is asymptotically stable;

(3) Let the conditions of Lemma $2.4(3)$ and $\mathfrak{f}_{3}<0$ hold. If $\mu_{1} \geq-\mathfrak{f}_{3} / \sigma_{2}$ or $\mu_{1}<$ $-\mathfrak{f}_{3} / \sigma_{2}$ and $\sigma_{1}>\bar{\delta}^{(3)}$, then the equilibrium $U_{3}^{*}$ of system (1.4) is asymptotically stable; If $\mu_{1}<-\mathfrak{f}_{3} / \sigma_{2}$ and $\sigma_{1}<\bar{\delta}^{(3)}$, then the equilibrium $U_{3}^{*}$ is unstable;

(4) Let the conditions of Lemma 2.4(3) or 2.4(4) and $\mathfrak{f}_{4}<0$ hold. If $\mu_{1} \geq-\mathfrak{f}_{4} / \sigma_{2}$ or $\mu_{1}<-\mathfrak{f}_{4} / \sigma_{2}$ and $\sigma_{1}>\bar{\delta}^{(4)}$, then the equilibrium $U_{4}^{*}$ of system (1.4) is asymptotically stable; If $\mu_{1}<-\mathfrak{f}_{4} / \sigma_{2}$ and $\sigma_{1}<\bar{\delta}^{(4)}$, then the equilibrium $U_{4}^{*}$ is unstable.

Proof. We only give the proof of (3) since the others can be proved similarly. The linearized operator of $H(U)$ at $U_{3}^{*}=\left(u_{3}^{*}, v_{3}^{*}\right)$ is

$$
H_{U}\left(U_{3}^{*}\right)=\left(\begin{array}{cc}
\sigma_{1} \Delta-u_{3}^{*} & -u_{3}^{*} \\
\mathfrak{f}_{3} & \sigma_{2} \Delta
\end{array}\right) .
$$

Following the Fourier series expansions similar to Section 2 , the eigenvalues of $H_{U}\left(U_{3}^{*}\right)$ are determined by the characteristic equation

$$
\beta^{2}-\operatorname{tr}\left(A_{j}\right) \beta+\operatorname{det}\left(A_{j}\right)=0, \quad j \geq 1,
$$

where

$$
\begin{gathered}
A_{j}=\left(\begin{array}{cc}
-\sigma_{1} \mu_{j}-u_{3}^{*} & -u_{3}^{*} \\
\mathfrak{f}_{3} & -\sigma_{2} \mu_{j}
\end{array}\right), \\
\operatorname{tr}\left(A_{j}\right)=-\left(\sigma_{1}+\sigma_{2}\right) \mu_{j}-u_{3}^{*}<0, \quad \operatorname{det}\left(A_{j}\right)=\sigma_{1} \sigma_{2} \mu_{j}^{2}+\sigma_{2} u_{3}^{*} \mu_{j}+\mathfrak{f}_{3} u_{3}^{*} .
\end{gathered}
$$

If $\mu_{1} \geq-\mathfrak{f}_{3} / \sigma_{2}$, then $\operatorname{det}\left(A_{j}\right)>0$ for all $j \geq 1$, which implies that $\operatorname{Re} \beta<0$ for all eigenvalues $\beta$. Hence, the equilibrium $U_{3}^{*}$ is asymptotically stable.

If $\mu_{1}<-\mathfrak{f}_{3} / \sigma_{2}$ and $\sigma_{1}>\bar{\delta}^{(3)}$, then $\mu_{j}<-\mathfrak{f}_{3} / \sigma_{2}$ for $j \in\left[1, j_{\tau}^{(3)}\right]$, and then we may suppose that the minimum of $\delta_{j}^{(3)}$ is attained at $m \in\left[1, j_{\tau}^{(3)}\right]$. Thus, we have $\sigma_{1}>\delta_{m}^{(3)}$, which implies $\operatorname{det}\left(A_{m}\right)>0$. Moreover, if $j>j_{\tau}^{(3)}$, then $\mu_{j} \geq-\mathfrak{f}_{3} / \sigma_{2}$, and then $\operatorname{det}\left(A_{j}\right)>0$. Therefore, the equilibrium $U_{3}^{*}$ is asymptotically stable.

Finally, if $\mu_{1}<-\mathfrak{f}_{3} / \sigma_{2}$ and $\sigma_{1}<\bar{\delta}^{(3)}$, then $\mu_{j}<-\mathfrak{f}_{3} / \sigma_{2}$ and $\sigma_{1}<\delta_{j}^{(3)}$ for $j \in\left[1, j_{\tau}^{(3)}\right]$. So $\operatorname{det}\left(A_{j}\right)<0$ for all $j \in\left[1, j_{\tau}^{(3)}\right]$. Therefore, the equilibrium $U_{3}^{*}$ is unstable in this case. 


\section{Long time behavior of solutions to system (1.4)}

Long time behavior of solutions to a system reflects the survival state of different substances or population in the system after a long time, which is an important part in studying of nonlinear dynamical systems. This section concentrates on the long time behavior of solutions to system (1.4) by applying the method in 12 .

Theorem 3.1. Let $q<(p+1)$. Then any solution $(u(x, t), v(x, t))$ of system (1.4) satisfies $\lim _{t \rightarrow \infty}(u(x, t), v(x, t))=(1,0)$ uniformly in $\bar{\Omega}$.

Proof. Suppose that $(u(x, t), v(x, t))$ is a solution of system (1.4). By the maximum principle and $u_{0}(x) \not \equiv 0$, we know that $u(x, t)>0, x \in \bar{\Omega}$. Let $z_{1}$ be a solution of system

$$
\left\{\begin{array}{l}
z_{1}^{\prime}(t)=z_{1}\left(1-z_{1}\right), \quad t>0, \\
z_{1}(0)=\max \left\{1, \max _{x \in \bar{\Omega}} u_{0}(x)\right\} .
\end{array}\right.
$$

Then $\lim _{t \rightarrow \infty} z_{1}(t)=1$. Moreover $u(x, t)$ satisfies

$$
\begin{cases}u_{t}-\sigma_{1} \Delta u \leq u(1-u), & x \in \Omega, t>0, \\ \frac{\partial u}{\partial \nu}=0, & x \in \partial \Omega, t>0, \\ u(x, 0)=u_{0}(x) \geq, \neq \equiv 0, & x \in \Omega .\end{cases}
$$

Then the comparison principle of parabolic equations induces that $u(x, t) \leq z_{1}(t)$ for all $x \in \bar{\Omega}$ and $t \geq 0$. Therefore

$$
\limsup _{t \rightarrow \infty} \max _{x \in \bar{\Omega}} u(x, t) \leq \lim _{t \rightarrow \infty} z_{1}(t)=1 .
$$

Consequently, for any small enough $\varepsilon>0$, there exists $T=T(\varepsilon) \gg 1$ such that $u(x, t) \leq$ $1+\varepsilon$ for all $x \in \bar{\Omega}$ and $t \geq T$. Thus $u(x, t) \leq \max \left\{1, \max _{x \in \bar{\Omega}} u_{0}(x)\right\}$ for $t \geq T$.

If $q<e(p+1)$ and $t \geq T$, then we have $\frac{q u}{p+u}-e \leq \frac{q}{p+1}-e<0$. Therefore, $v(x, t)$ satisfies

$$
\begin{cases}v_{t}-\sigma_{2} \Delta v \leq\left(\frac{q}{p+1}-e\right) v, & x \in \Omega, t>T \\ \frac{\partial v}{\partial \nu}=0, & x \in \partial \Omega, t>T \\ v(x, t)>0, & x \in \Omega, t=T .\end{cases}
$$

This shows that $v$ is a lower solution of the problem

$$
\begin{cases}z_{2}^{\prime}(t)-\sigma_{2} \Delta z_{2}=\left(\frac{q}{p+1}-e\right) z_{2}, & x \in \Omega, t>T, \\ \frac{\partial z_{2}}{\partial \nu}=0, & x \in \partial \Omega, t>T, \\ z_{2}=\max _{x \in \bar{\Omega}} v(x, T), & x \in \Omega .\end{cases}
$$


Moreover, it is easy to see that the function $C \exp \left\{\left(\frac{q}{p+1}-e\right) t\right\}$ is a positive upper solution of 3.1 for constant $C>\max _{\bar{\Omega}} v(x, T)$. By the comparison principle of parabolic equations, we obtain that $v(x, t) \leq C \exp \left\{\left(\frac{q}{p+1}-e\right) t\right\}$ for all $x \in \bar{\Omega}$ and $t \geq T$. Therefore, we have

$$
\limsup _{t \rightarrow \infty} \max _{x \in \bar{\Omega}} v(x, t) \leq \lim _{t \rightarrow \infty} C \exp \left\{\left(\frac{q}{p+1}-e\right) t\right\}=0 .
$$

Consequently, $\lim _{t \rightarrow \infty} v(x, t)=0$ for $x \in \bar{\Omega}$. Next, we show that $\lim _{t \rightarrow \infty} u(x, t)=1$.

For some large $t_{1}>T$. Let $z_{3}$ be a solution of system

$$
\left\{\begin{array}{l}
z_{3}^{\prime}(t)=z_{3}\left(1-z_{3}\right)-C \exp \left\{\left(\frac{q}{p+1}-e\right) t\right\} z_{3}, \quad t>t_{1}, \\
z_{3}\left(t_{1}\right)=\frac{1}{2} \min \left\{1, \min _{x \in \bar{\Omega}} u\left(x, t_{1}\right)\right\}>0 .
\end{array}\right.
$$

Then $\lim _{t \rightarrow \infty} z_{3}(t)=1$ when $q<e(p+1)$. Moreover, $u(x, t)$ also satisfies

$$
\begin{cases}u_{t}-\sigma_{1} \Delta u \geq u(1-u)-C \exp \left\{\left(\frac{q}{p+1}-e\right) t\right\} u, & x \in \Omega, t>t_{1}, \\ \frac{\partial u}{\partial \nu}=0, & x \in \partial \Omega, t>t_{1}, \\ u\left(x, t_{1}\right)>0, & x \in \Omega .\end{cases}
$$

Then the comparison principle of parabolic equations implies that $u(x, t) \geq z_{3}(t)$ for all $x \in \bar{\Omega}$ and $t \geq t_{1}$. Thus

$$
\liminf _{t \rightarrow \infty} \min _{x \in \bar{\Omega}} u(x, t) \geq \lim _{t \rightarrow \infty} z_{3}(t)=1, \quad x \in \bar{\Omega} .
$$

Therefore, $\lim _{t \rightarrow \infty} u(x, t)=1$. The proof is complete.

Remark 3.2. Theorem 3.1 means that tumour cells will only exist in the body when the steepness $p$ of immune response and the death rate $e$ of effector cells are fixed and the maximum immune response rate $q$ is small. Moreover, we see from $\lim _{t \rightarrow \infty} u(x, t)=1$ that the concentration of tumour cells does not increase indefinitely, but tends to stabilize over time.

\section{Bifurcation analysis of the elliptic system}

Let $\Omega=(0, l \pi), l \in \mathbb{R}^{+}$. Then the steady-state problem corresponding to $(1.4)$ is given by

$$
\left\{\begin{aligned}
-\sigma_{1} u^{\prime \prime} & =u(1-u)-u v, & & x \in(0, l \pi), \\
-\sigma_{2} v^{\prime \prime} & =\frac{q u v}{p+u}-s u v-e v, & & x \in(0, l \pi), \\
u^{\prime}=v^{\prime} & =0, & & x=0, l \pi
\end{aligned}\right.
$$

Clearly, system 4.1 has equilibria $U_{i}^{*}, i=1,2,3,4$, which also possess the result of stability as Theorem 2.5. Notice that a steady state bifurcation solution of system (1.4) 
in the one-dimensional interval $\Omega=(0, l \pi)(l>0)$ is a non-constant positive solution of system 4.1). In this section, by taking tumour diffusion rate $\sigma_{1}$ as a parameter and using the Crandall-Rabinowitz bifurcation theory [6], we discuss the existence of local steadystate bifurcation emitting from positive equilibria $U_{3}^{*}$ and $U_{4}^{*}$ of system (4.1). Then we formulate a global steady-state bifurcation of system 4.1) by using the global bifurcation theory 26]. Finally, the stability of bifurcating solution is analyzed.

It is well known that the eigenvalue problem

$$
-u^{\prime \prime}=\mu u, \quad x \in(0, l \pi), \quad u^{\prime}(x)=0, \quad x=0, l \pi
$$

has eigenvalues $\mu_{j}=j^{2} / l^{2}, j=0,1, \ldots$ with corresponding normalized eigenfunctions

$$
\varphi_{j}(x)= \begin{cases}\frac{1}{\sqrt{l \pi}}, & j=0, \\ \sqrt{\frac{2}{l \pi}} \cos \frac{j x}{l}, & j>0,\end{cases}
$$

where the characteristic functions $\left\{\varphi_{j}\right\}_{j=0}^{\infty}$ form an normalized orthonormal basis in $L^{2}(0, l \pi)$.

Let $Y=L^{2}(0, l \pi) \times L^{2}(0, l \pi)$ and denote by $\left(V_{1}, V_{2}\right)_{Y}=\left(u_{1}, u_{2}\right)_{L^{2}(0, l \pi)}+\left(v_{1}, v_{2}\right)_{L^{2}(0, l \pi)}$ the inner product in $Y$ with $V_{1}=\left(u_{1}, v_{1}\right), V_{2}=\left(u_{2}, v_{2}\right) \in Y$. Set $X=\{(u, v) \mid u, v \in$ $\left.C^{2}([0, l \pi]), u^{\prime}=v^{\prime}=0, x=0, l \pi\right\}$. Define the map $E:(0,+\infty) \times X \rightarrow Y$ as

$$
E\left(\sigma_{1}, U\right)=\left(\sigma_{1} u^{\prime \prime}+u(1-u)-u v, \sigma_{2} v^{\prime \prime}+\frac{q u v}{p+u}-s u v-e v\right)^{T}, \quad U=(u, v)^{T} .
$$

Then the solution of the system (4.1) is equivalent to the zero point of $E$. Note that $E\left(\sigma_{1}, U_{3}^{*}\right)=E\left(\sigma_{1}, U_{4}^{*}\right)=0$ for any $\sigma_{1}>0$.

Now, we discuss the positive solutions of the system 4.1) emitting from positive equilibrium $U_{3}^{*}$. The main conclusion reads as the followings.

Theorem 4.1. Assume that the conditions of Lemma 2.4(3) and $\mathfrak{f}_{3}<0$ hold. Let there be a positive integer $j$ such that $\mu_{j} \sigma_{2}<-\mathfrak{f}_{3}$. If $\delta_{i}^{(3)} \neq \delta_{j}^{(3)}$ for any $i \neq j$ and $i, j \in\left[1, j_{\tau}^{(3)}\right]$, then $\left(\delta_{j}^{(3)}, U_{3}^{*}\right)$ is a bifurcation point of $E=0$. Moreover, there exists a one-parameter family of nonconstant solutions $\left(\sigma_{1}(\vartheta), U(\vartheta)\right)$ of the system 4.1 for $|\vartheta|$ sufficiently small, where

$$
U(\vartheta)=(u(\vartheta), v(\vartheta))=\left(u_{3}^{*}+\vartheta \varphi_{j}+\vartheta \phi(\vartheta), v_{3}^{*}+\vartheta m_{j} \varphi_{j}+\vartheta \psi(\vartheta)\right)
$$

$\sigma_{1}(0)=\delta_{j}^{(3)}, U(0)=(u(0), v(0))=U_{3}^{*}, \sigma_{1}(\vartheta), u(\vartheta), v(\vartheta)$ are continuous functions with respect to $\vartheta, \phi(\vartheta), \psi(\vartheta) \in\left\{\operatorname{ker}\left(E_{U}\left(\delta_{j}^{(3)}, U_{3}^{*}\right)\right)\right\}^{\perp}$ and $m_{j}=\mathfrak{f}_{3} /\left(\sigma_{2} \mu_{j}\right)$.

Proof. The Fréchet derivative of $E$ at $U_{3}^{*}$ can be expressed by

$$
J\left(\sigma_{1}\right)=E_{U}\left(\sigma_{1}, U_{3}^{*}\right)=\left(\begin{array}{cc}
\sigma_{1} \frac{d^{2}}{d x^{2}}-u_{3}^{*} & -u_{3}^{*} \\
\mathfrak{f}_{3} & \sigma_{2} \frac{d^{2}}{d x^{2}}
\end{array}\right)
$$


It is clear that the linear operators $E_{U}, E_{\sigma_{1}}$ and $E_{U \sigma_{1}}$ are continuous.

According to the assumptions, we have $\operatorname{ker}\left(J\left(\delta_{j}^{(3)}\right)\right)=\operatorname{span}\{\Phi\}$, where

$$
\Phi=\left(\begin{array}{c}
1 \\
m_{j}
\end{array}\right) \varphi_{j}, \quad m_{j}=\frac{\mathfrak{f}_{3}}{\sigma_{2} \mu_{j}}<0 .
$$

Denote by $J^{*}\left(\delta_{j}^{(3)}\right)$ the conjugate operator of $J\left(\delta_{j}^{(3)}\right)$. Then a similar computation gives $\operatorname{ker}\left(J^{*}\left(\delta_{j}^{(3)}\right)\right)=\operatorname{span}\left\{\Phi^{*}\right\}$ with

$$
\Phi^{*}=\left(\begin{array}{c}
1 \\
m_{j}^{*}
\end{array}\right) \varphi_{j}, \quad m_{j}^{*}=-\frac{u_{3}^{*}}{\sigma_{2} \mu_{j}}<0 .
$$

By the Fredholm alternative theorem, we have $R\left(J\left(\delta_{j}^{(3)}\right)\right)=\left\{\operatorname{ker}\left(J^{*}\left(\delta_{j}^{(3)}\right)\right)\right\}^{\perp}$. Therefore $\operatorname{codim}\left(R\left(J\left(\delta_{j}^{(3)}\right)\right)\right)=\operatorname{dim}\left(\operatorname{ker}\left(J^{*}\left(\delta_{j}^{(3)}\right)\right)\right)=1$. Furthermore, since

$$
J_{\sigma_{1}}\left(\delta_{j}^{(3)}\right) \Phi=\left(\begin{array}{cc}
\frac{d^{2}}{d x^{2}} & 0 \\
0 & 0
\end{array}\right) \Phi=\left(\begin{array}{c}
-\mu_{j} \varphi_{j} \\
0
\end{array}\right)
$$

and $\left(J_{\sigma_{1}}\left(\delta_{j}^{(3)}\right) \Phi, \Phi^{*}\right)_{Y}=\left(-\mu_{j} \varphi_{j}, \varphi_{j}\right)_{L^{2}(0, l \pi)}<0, J_{\sigma_{1}}\left(\delta_{j}^{(3)}\right) \Phi \notin R\left(J\left(\delta_{j}^{(3)}\right)\right)$. By the CrandallRabinowitz bifurcation theorem, we see that Theorem 4.1 holds.

Remark 4.2. The assumption $\delta_{i}^{(3)} \neq \delta_{j}^{(3)}$ for any $i \neq j$ is crucial in Theorem 4.1, which guarantees $\operatorname{dim}\left(\operatorname{ker}\left(J\left(\delta_{j}^{(3)}\right)\right)\right)=1$ and the Crandall-Rabinowitz bifurcation theorem emitting from simple eigenvalue can be applied. In fact, from (2.4) we know that $\delta_{j}^{(3)}=$ $\frac{-u_{3}^{*} \sigma_{2} \mu_{j}-\mathfrak{f}_{3} u_{3}^{*}}{\sigma_{2} \mu_{j}^{2}}$. Further, let $\mathfrak{f}_{3}<0$. Then we can simply calculate that $\delta_{j}^{(3)}$ is decreasing with respect to $\mu_{j}$ when $\mu_{j}<-2 \mathfrak{f}_{3} / \sigma_{2}$ and increasing with respect to $\mu_{j}$ when $\mu_{j}>-2 \mathfrak{f}_{3} / \sigma_{2}$. And since $\delta_{j}^{(3)}>0$ when $\mu_{j}<-\mathfrak{f}_{3} / \sigma_{2}$, under the condition that the biological meaning is satisfied (i.e., $\left.\mu_{j}<-\mathfrak{f}_{3} / \sigma_{2}\right), \delta_{j}^{(3)}$ is decreasing with respect to $\mu_{j}$. This means that system 4.1 has only simple bifurcation point, and the eigenvalue $\mu_{j}$ is simple in this case. Due to $\mu_{j}<-\mathfrak{f}_{3} / \sigma_{2}$ implies that $\sigma_{2}<-\mathfrak{f}_{3} / \mu_{j}$, so the tumour cells and effector cells can coexist for small diffusion rate $\sigma_{2}$ of effector cells.

Let $\Gamma_{j}=\left(\sigma_{1}(\vartheta), u(\vartheta), v(\vartheta)\right)$. It is clear that Theorem 4.1 only provides the local structure of the bifurcation curve $\Gamma_{j}$, but does not present the information when the bifurcation curve $\Gamma_{j}$ is far from the equilibrium. Thus, the next part focuses on the global structure of bifurcation curve $\Gamma_{j}$. For that purpose, we first give a priori estimate of the system 4.1), which is useful in proving the existence of the global bifurcation of the system 4.1. 
Theorem 4.3. Assume that $q>s$ and $p>1$. Then any nonnegative solution $(u(x), v(x))$ of system 4.1 satisfies

$$
0 \leq u(x) \leq 1, \quad 0 \leq v(x) \leq\left(\frac{1}{4 e}+\frac{\sigma_{1}}{\sigma_{2}}\right)(q-s), \quad x \in[0, l \pi] .
$$

Proof. By the maximum principle, we have $u(x) \leq 1$ for all $x \in[0, l \pi]$. Multiplying the first equation of (4.1) by $q-s$ and then adding it to the second equation, we get

$$
\begin{aligned}
-\frac{\partial^{2}}{\partial x^{2}}\left[\sigma_{1}(q-s) u+\sigma_{2} v\right] & =(q-s) u(1-u)-(q-s) u v+\frac{q u v}{p+u}-s u v-e v \\
& \leq(q-s) u(1-u)-(q-s) u v+(q-s) u v-e v \\
& =(q-s) u(1-u)-e v \\
& =(q-s) u(1-u)+\frac{e \sigma_{1}}{\sigma_{2}}(q-s) u-\frac{e \sigma_{1}}{\sigma_{2}}(q-s) u-\frac{e \sigma_{2}}{\sigma_{2}} v \\
& \leq\left(\frac{1}{4}+\frac{e \sigma_{1}}{\sigma_{2}}\right)(q-s)-\frac{e}{\sigma_{2}}\left[\sigma_{1}(q-s) u+\sigma_{2} v\right] .
\end{aligned}
$$

Using the maximum principle again, we have $\sigma_{1}(q-s) u+\sigma_{2} v \leq\left(\frac{\sigma_{2}}{4 e}+\sigma_{1}\right)(q-s)$, then $v(x) \leq\left(\frac{1}{4 e}+\frac{\sigma_{1}}{\sigma_{2}}\right)(q-s)$. The proof is accomplished.

Theorem 4.4. (1) Suppose that the assumptions of Theorem 4.1 hold. Then the bifurcation curve $\Gamma_{j}$ of the nonconstant solutions of system (4.1) bifurcating from $\left(\delta_{j}^{(3)}, U_{3}^{*}\right)$ is contained in a global branch $\Sigma_{j}$ of the nonconstant solutions of system 4.1.

(2) Either $\bar{\Sigma}_{j}$ contains another bifurcation point $\left(\delta_{k}^{(3)}, U_{3}^{*}\right)$ for $k \neq j$, or the projection of $\bar{\Sigma}_{j}$ onto $\sigma_{1}$-axis contains the interval $\left(0, \delta_{j}^{(3)}\right)$.

Proof. Let $\chi=u-u_{3}^{*}, \gamma=v-v_{3}^{*}$. Then we define a nonlinear equation

$$
F\left(\sigma_{1},\left(\begin{array}{l}
\chi \\
\gamma
\end{array}\right)\right)=\left(\begin{array}{c}
\sigma_{1} \chi^{\prime \prime}-u_{3}^{*} \chi-u_{3}^{*} \gamma-\chi^{2}-\chi \gamma \\
\sigma_{2} \gamma^{\prime \prime}+\left(\gamma+v_{3}^{*}\right)\left(\frac{q\left(\chi+u_{3}^{*}\right)}{p+\chi+u_{3}^{*}}-s\left(\chi+u_{3}^{*}\right)-e\right)
\end{array}\right)
$$

Define

$$
Z=\left\{\left(\sigma_{1},\left(\begin{array}{l}
\chi \\
\gamma
\end{array}\right)\right):(\chi, \gamma) \in X \text { and } \chi+u_{3}^{*} \geq 0, \gamma+v_{3}^{*} \geq 0\right\}
$$

Then $\left\{\left(\sigma_{1}, 0,0\right)\right\}$ is a line of trivial solutions for $F=0$, and then Theorem 4.3 in 26 can be applied to each continuum $\Sigma_{j}$ bifurcating from $\left(\delta_{j}^{(3)}, 0,0\right)$. For each continuum $\Sigma_{j}$, either $\bar{\Sigma}_{j}$ contains $\left(\delta_{k}^{(3)}, 0,0\right), \delta_{k}^{(3)} \neq \delta_{j}^{(3)}$ with $\delta_{k}^{(3)}$ being another bifurcation value, or $\Sigma_{j}$ is not compact.

From Theorem 4.3. we know that any solution $(u(x), v(x))$ of system 4.1 is bounded in $L^{\infty}(\Omega)$, then it is also bounded in $X$ in view of $L^{p}$ estimate and Schauder estimate. Therefore, if $\Sigma_{j}$ is not compact, then the projection of $\bar{\Sigma}_{j}$ onto $\sigma_{1}$-axis contains $\left(0, \delta_{j}^{(3)}\right)$. 
Similarly, we can get the structure of the bifurcation solution of system 4.1 emitting from $U_{4}^{*}$.

Theorem 4.5. (1) Assume that the conditions of Lemma 2.4(4) and $\mathfrak{f}_{4}<0$ hold. Let there be a positive integer $j$ such that $\mu_{j} \sigma_{2}<-\mathfrak{f}_{4}$. If $\delta_{i}^{(4)} \neq \delta_{j}^{(4)}$ for any $i \neq j$ and $i, j \in\left[1, j_{\tau}^{(4)}\right]$, then $\left(\delta_{j}^{(4)}, U_{4}^{*}\right)$ is a bifurcation point of $E=0$. Moreover, there exists a one-parameter family of nonconstant solutions $\Upsilon_{j}=\left(\bar{\sigma}_{1}(\vartheta), \bar{U}(\vartheta)\right)$ of system (4.1) for $|\vartheta|$ sufficiently small, where $\sigma_{1}(0)=\delta_{j}^{(4)}, \bar{U}(0)=(\bar{u}(0), \bar{v}(0))=U_{4}^{*}, \bar{U}(\vartheta)=(\bar{u}(\vartheta), \bar{v}(\vartheta))=$ $\left(u_{4}^{*}+\vartheta \varphi_{j}+\vartheta \bar{\phi}(\vartheta), v_{4}^{*}+\vartheta h_{j} \varphi_{j}+\vartheta \bar{\psi}(\vartheta)\right), \bar{\sigma}_{1}(\vartheta), \bar{u}(\vartheta), \bar{v}(\vartheta)$ are continuous functions with respect to $\vartheta, \bar{\phi}(\vartheta), \bar{\psi}(\vartheta) \in\left\{\operatorname{ker}\left(E_{U}\left(\delta_{j}^{(4)}, U_{4}^{*}\right)\right)\right\}^{\perp}$ and $h_{j}=\mathfrak{f}_{4} /\left(\sigma_{2} \mu_{j}\right)$.

(2) Under Theorem 4.5(1), the bifurcation curve $\Upsilon_{j}$ of the nonconstant solutions of system (4.1) bifurcating from $\left(\delta_{j}^{(4)}, U_{4}^{*}\right)$ are contained in a global branch $\Pi_{j}$ of the nonconstant solution of system (4.1). Either $\bar{\Pi}_{j}$ contains another bifurcation point $\left(\delta_{k}^{(4)}, U_{4}^{*}\right)$ for $k \neq j$, or the projection of $\bar{\Pi}_{j}$ onto $\sigma_{1}$-axis contains the interval $\left(0, \delta_{j}^{(4)}\right)$.

In the following, we consider the stability of the bifurcation solution $U(\vartheta)$ obtained above. The stability of $\bar{U}(\vartheta)$ can be proved similarly, we omit it. Let

$$
G\left(u_{3}^{*}, v_{3}^{*}\right)=-\frac{4 q p v_{3}^{*}}{\left(p+u_{3}^{*}\right)^{3}} \phi^{\prime}(0)+2\left(-s+\frac{q p}{\left(p+u_{3}^{*}\right)^{2}}\right)\left(\psi^{\prime}(0)+m_{j} \phi^{\prime}(0)\right) .
$$

Then the following result can be obtained.

Theorem 4.6. Suppose that Theorem 4.1 holds. If $\mathfrak{f}_{3}^{-1} u_{3}^{*} m_{j} G\left(u_{3}^{*}, v_{3}^{*}\right)+\phi^{\prime}(0)>0$, then the bifurcation solution $U(\vartheta)$ of system (4.1) is stable; If $\mathfrak{f}_{3}^{-1} u_{3}^{*} m_{j} G\left(u_{3}^{*}, v_{3}^{*}\right)+\phi^{\prime}(0)<0$, then $U(\vartheta)$ is unstable.

Proof. Denote by $E_{U}\left(\sigma_{1}, U_{3}^{*}\right)$ and $E_{U}\left(\sigma_{1}(\vartheta), U(\vartheta)\right)$ the variational matrices of the steadystate problem 4.1) at $\left(\sigma_{1}, U_{3}^{*}\right)$ and $\left(\sigma_{1}(\vartheta), U(\vartheta)\right)$, respectively. Set $V_{0}=\left(\varphi_{j}, m_{j} \varphi_{j}\right)$, $V_{0}^{*}=\left(\varphi_{j}, m_{j}^{*} \varphi_{j}\right)$. Since $\left(i V_{0}, V_{0}^{*}\right)_{L^{2}(0, l \pi)}=1+m_{j} m_{j}^{*}=1-\frac{\mathfrak{f}_{3} u_{3}^{*}}{\left(\sigma_{2} \mu_{j}\right)^{2}}>0,0$ is an $i$-simple eigenvalue of $E\left(\delta_{j}^{(3)}, U_{3}^{*}\right)$ by the Definition 13.6 in [27. According to Lemma 13.7 in [27], there exist smooth functions $\sigma_{1} \rightarrow \rho\left(\sigma_{1}\right)$ and $\vartheta \rightarrow \varrho(\vartheta)$ in the neighbourhoods of $\delta_{j}^{(3)}$ and 0 respectively such that $\rho\left(\sigma_{1}\right), \varrho(\vartheta)$ are the eigenvalues of $E_{U}\left(\sigma_{1}, U_{3}^{*}\right)$ and $E_{U}\left(\sigma_{1}(\vartheta), U(\vartheta)\right)$ respectively, and $\rho\left(\sigma_{1}\right), \varrho(\vartheta)$ satisfy $\rho\left(\delta_{j}^{(3)}\right)=0=\varrho(0), \rho^{\prime}\left(\delta_{j}^{(3)}\right) \neq 0$. So, there exists $j>0$ such that

$$
\rho^{2}+\left[u_{3}^{*}+\left(\sigma_{1}+\sigma_{2}\right) \mu_{j}\right] \rho+\sigma_{1} \sigma_{2} \mu_{j}^{2}+\sigma_{2} u_{3}^{*} \mu_{j}+\mathfrak{f}_{3} u_{3}^{*}=0
$$

Differentiating on $\sigma_{1}$ and then taking $\sigma_{1}=\delta_{j}^{(3)}$, we have

$$
\rho^{\prime}\left(\delta_{j}^{(3)}\right)=-\frac{\sigma_{2} \mu_{j}^{2}}{u_{3}^{*}+\left(\sigma_{1}+\sigma_{2}\right) \mu_{j}}<0 .
$$


Then Theorem 13.8 in 27 implies that

$$
\lim _{\vartheta \rightarrow 0} \frac{\vartheta \rho^{\prime}\left(\delta_{j}^{(3)}\right) \sigma_{1}^{\prime}(\vartheta)}{\varrho(\vartheta)}=-1
$$

It is easy to see from (4.4) that the sign of $\varrho(\vartheta)$ is determined by $\sigma_{1}^{\prime}(\vartheta)$. Next, we investigate the sign of $\sigma_{1}^{\prime}(\vartheta)$.

Set $F_{1}(u, v)=-u^{2}-u v, F_{2}(u, v)=\frac{q u v}{p+u}-s u v$. Then by substituting $u(\vartheta)=u_{3}^{*}+$ $\vartheta \varphi_{j}+\vartheta \phi(\vartheta), v(\vartheta)=v_{3}^{*}+\vartheta m_{j} \varphi_{j}+\vartheta \psi(\vartheta)$ into (4.1), we get

$$
\begin{aligned}
& \sigma_{1}(\vartheta) \frac{d^{2}}{d x^{2}}\left(u_{3}^{*}+\vartheta\left(\varphi_{j}+\phi(\vartheta)\right)\right)+u_{3}^{*}+\vartheta\left(\varphi_{j}+\phi(\vartheta)\right) \\
+ & F_{1}\left(u_{3}^{*}+\vartheta\left(\varphi_{j}+\phi(\vartheta)\right), v_{3}^{*}+\vartheta\left(m_{j} \varphi_{j}+\psi(\vartheta)\right)\right)=0, \\
& \sigma_{2} \frac{d^{2}}{d x^{2}}\left(v_{3}^{*}+\vartheta\left(m_{j} \varphi_{j}+\psi(\vartheta)\right)\right)-e\left(v_{3}^{*}+\vartheta\left(m_{j} \varphi_{j}+\psi(\vartheta)\right)\right) \\
+ & F_{2}\left(u_{3}^{*}+\vartheta\left(\varphi_{j}+\phi(\vartheta)\right), v_{3}^{*}+\vartheta\left(m_{j} \varphi_{j}+\psi(\vartheta)\right)\right)=0 .
\end{aligned}
$$

Substituting the Taylor series of $F_{i}\left(u_{3}^{*}+\vartheta\left(\varphi_{j}+\phi(\vartheta)\right), v_{3}^{*}+\vartheta\left(m_{j} \varphi_{j}+\psi(\vartheta)\right)\right), i=1,2$ into (4.5), then dividing by and differentiating on $\vartheta$ in turn, and then taking $\vartheta=0$, we have

$$
\begin{cases}-\sigma_{1}^{\prime}(0) \mu_{j} \varphi_{j}+\sigma_{1}(0) \frac{d^{2}\left(\phi^{\prime}(0)\right)}{d x^{2}}-u_{3}^{*} \phi^{\prime}(0)-u_{3}^{*} \psi^{\prime}(0)-\frac{1}{2} \varphi_{j}^{2}=0, & x \in(0, l \pi), \\ \sigma_{2} \frac{d^{2}\left(\psi^{\prime}(0)\right)}{d x^{2}}+\mathfrak{f}_{3} \phi^{\prime}(0)+\left(\frac{q p\left(m_{j}-v_{3}^{*}\right)}{\left(p+u_{3}^{*}\right)^{2}}-s\right) \varphi_{j}^{2}=0, & x \in(0, l \pi) .\end{cases}
$$

Multiplying the two equations of (4.6) by $\varphi_{j}$ and $m_{j} \varphi_{j}$ respectively, then integrating over $(0, l \pi)$, we get

$$
\begin{aligned}
\sigma_{1}^{\prime}(0) \mu_{j} \int_{0}^{l \pi} \varphi_{j}^{2} \mathrm{~d} x= & \int_{0}^{l \pi}\left[\sigma_{1}(0) \frac{d^{2}\left(\phi^{\prime}(0)\right)}{d x^{2}}-u_{3}^{*} \phi^{\prime}(0)-u_{3}^{*} m_{j} \phi^{\prime}(0)\right] \varphi_{j} \mathrm{~d} x \\
& +\int_{0}^{l \pi} u_{3}^{*}\left[m_{j} \phi^{\prime}(0)-\psi^{\prime}(0)\right] \varphi_{j} \mathrm{~d} x-\frac{1}{2} \int_{0}^{l \pi} \varphi_{j}^{3} \mathrm{~d} x \\
= & \int_{0}^{l \pi} u_{3}^{*}\left[m_{j} \phi^{\prime}(0)-\psi^{\prime}(0)\right] \varphi_{j} \mathrm{~d} x-\frac{1}{2} \int_{0}^{l \pi} \varphi_{j}^{3} \mathrm{~d} x
\end{aligned}
$$

and

$$
\begin{aligned}
& \int_{0}^{l \pi}\left[\sigma_{2} m_{j} \frac{d^{2}\left(\psi^{\prime}(0)\right)}{d x^{2}}+\mathfrak{f}_{3} \psi^{\prime}(0)\right] \varphi_{j} \mathrm{~d} x+\int_{0}^{l \pi} \mathfrak{f}_{3}\left[m_{j} \phi^{\prime}(0)-\psi^{\prime}(0)\right] \varphi_{j} \mathrm{~d} x \\
& +\left(\frac{q p\left(m_{j}-v_{3}^{*}\right)}{\left(p+u_{3}^{*}\right)^{2}}-s\right) \int_{0}^{l \pi} \varphi_{j}^{3} \mathrm{~d} x \\
= & \int_{0}^{l \pi} \mathfrak{f}_{3}\left[m_{j} \phi^{\prime}(0)-\psi^{\prime}(0)\right] \varphi_{j} \mathrm{~d} x+\left(\frac{q p\left(m_{j}-v_{3}^{*}\right)}{\left(p+u_{3}^{*}\right)^{2}}-s\right) \int_{0}^{l \pi} \varphi_{j}^{3} \mathrm{~d} x=0 .
\end{aligned}
$$


By 4.7) and 4.8, we obtain

$$
\sigma_{1}^{\prime}(0) \mu_{j} \int_{0}^{l \pi} \varphi_{j}^{2} \mathrm{~d} x=-\left[\mathfrak{f}_{3}^{-1} u_{3}^{*}\left(\frac{q p\left(m_{j}-v_{3}^{*}\right)}{\left(p+u_{3}^{*}\right)^{2}}-s\right)+\frac{1}{2}\right] \int_{0}^{l \pi} \varphi_{j}^{3} \mathrm{~d} x
$$

SO

$$
\sigma_{1}^{\prime}(0)=\frac{-\left[\mathfrak{f}_{3}^{-1} u_{3}^{*}\left(\frac{q p\left(m_{j}-v_{3}^{*}\right)}{\left(p+u_{3}^{*}\right)^{2}}-s\right)+\frac{1}{2}\right] \int_{0}^{l \pi} \varphi_{j}^{3} \mathrm{~d} x}{\mu_{j} \int_{0}^{l \pi} \varphi_{j}^{2} \mathrm{~d} x} .
$$

By the definition of $\varphi_{j}$ in 4.2 , we know that $\int_{0}^{l \pi} \varphi_{j}^{2} \mathrm{~d} x=1, \int_{0}^{l \pi} \varphi_{j}^{3} \mathrm{~d} x=0$ for $j \neq 0$, so $\sigma_{1}^{\prime}(0)=0$. Consequently, for small $\vartheta>0$, the sign of $\sigma_{1}^{\prime}(\vartheta)$ is unknown, and further, the sign of $\varrho(\vartheta)$ is uncertain, which leads to the stability of $U(\vartheta)$ is also unknown. In this case, it needs to change the idea to consider the stability of $U(\vartheta)$. Here we endow $\sigma_{1}(\vartheta)$ with stronger smoothness.

Next, we calculate the sign of $\sigma_{1}^{\prime \prime}(0)$, which is given by

$$
\begin{aligned}
\sigma_{1}^{\prime \prime}(0) \mu_{j} \int_{0}^{l \pi} \varphi_{j}^{2} \mathrm{~d} x= & \int_{0}^{l \pi}\left[\sigma_{1}(0) \frac{d^{2}\left(\phi^{\prime \prime}(0)\right)}{d x^{2}}-u_{3}^{*} \phi^{\prime \prime}(0)-u_{3}^{*} m_{j} \phi^{\prime \prime}(0)\right] \varphi_{j} \mathrm{~d} x \\
& +\int_{0}^{l \pi} u_{3}^{*}\left[m_{j} \phi^{\prime \prime}(0)-\psi^{\prime \prime}(0)\right] \varphi_{j} \mathrm{~d} x-\int_{0}^{l \pi} \phi^{\prime}(0) \varphi_{j}^{2} \mathrm{~d} x \\
= & \int_{0}^{l \pi} u_{3}^{*}\left[m_{j} \phi^{\prime \prime}(0)-\psi^{\prime \prime}(0)\right] \varphi_{j} \mathrm{~d} x-\int_{0}^{l \pi} \phi^{\prime}(0) \varphi_{j}^{2} \mathrm{~d} x .
\end{aligned}
$$

Furthermore, we have

$$
\begin{aligned}
& \int_{0}^{l \pi}\left[\sigma_{2} m_{j} \frac{d^{2}\left(\psi^{\prime \prime}(0)\right)}{d x^{2}}+\mathfrak{f}_{3} \psi^{\prime \prime}(0)\right] \varphi_{j} \mathrm{~d} x+\int_{0}^{l \pi} \mathfrak{f}_{3}\left[m_{j} \phi^{\prime \prime}(0)-\psi^{\prime \prime}(0)\right] \varphi_{j} \mathrm{~d} x \\
& +\int_{0}^{l \pi} G\left(u_{3}^{*}, v_{3}^{*}\right) m_{j} \varphi_{j}^{2} \mathrm{~d} x \\
= & \int_{0}^{l \pi} \mathfrak{f}_{3}\left[m_{j} \phi^{\prime \prime}(0)-\psi^{\prime \prime}(0)\right] \varphi_{j} \mathrm{~d} x+\int_{0}^{l \pi} G\left(u_{3}^{*}, v_{3}^{*}\right) m_{j} \varphi_{j}^{2} \mathrm{~d} x=0
\end{aligned}
$$

with $G\left(u_{3}^{*}, v_{3}^{*}\right)=-\frac{4 q p v_{3}^{*}}{\left(p+u_{3}^{*}\right)^{3}} \phi^{\prime}(0)+2\left(-s+\frac{q p}{\left(p+u_{3}^{*}\right)^{2}}\right)\left(\psi^{\prime}(0)+m_{j} \phi^{\prime}(0)\right)$. Combining 4.9) with 4.10, we have

$$
\sigma_{1}^{\prime \prime}(0)=\frac{\int_{0}^{l \pi}\left[-\mathfrak{f}_{3}^{-1} u_{3}^{*} m_{j} G\left(u_{3}^{*}, v_{3}^{*}\right)-\phi^{\prime}(0)\right] \varphi_{j}^{2} \mathrm{~d} x}{\mu_{j}} .
$$

Therefore, $\sigma_{1}^{\prime \prime}(0)>(<) 0$ if $\mathfrak{f}_{3}^{-1} u_{3}^{*} m_{j} G\left(u_{3}^{*}, v_{3}^{*}\right)+\phi^{\prime}(0)<(>) 0$, and hence, $\sigma_{1}^{\prime}(\vartheta)>(<) 0$. Combining (4.3) with 4.4), Theorem 4.6 follows.

Remark 4.7. Theorems 4.1, 4.4 and 4.5 prove that system 4.1 exists steady-state bifurcation solution, Theorem 4.6 shows that the bifurcation solution is stable when $\mathfrak{f}_{3}^{-1} u_{3}^{*} m_{j}$ $G\left(u_{3}^{*}, v_{3}^{*}\right)+\phi^{\prime}(0)>0$ holds. Biologically, these mean that tumour cells and effector cells can coexist. However, tumour cells cannot increase infinitely since the concentration of tumour cells tends to be stable over time. 


\section{Numerical simulations}

Numerical analysis provides a more intuitive way for us to understand biological systems. Thus, in order to better understand the characteristics and interactions of species in biological systems, it is necessary to combine theoretical analysis with numerical calculation. In this section, we give some numerical examples to support and supplement our theoretical analysis.

Firstly, we consider the stability of trivial equilibrium $U_{0}=(0,0)$ corresponding to systems (1.3) and (1.4), respectively. Especially, for the convenience of calculation, we only consider one-dimensional case, that is, $\Omega=(0, l \pi)$.

For system 1.3 , let $l=1, \sigma_{2}=1.5, p=0.1, s=0.1, q=3.2, e=0.2$. Then $\mu_{0}=1$, and we know from Theorem 2.1 $(1)$ that the trivial equilibrium $U_{0}=(0,0)$ is locally asymptotically stable when $\sigma_{1}=2>1 / \mu_{0}=1$ (see Figure 5.1) and unstable when $\sigma_{1}=0.2<1 / \mu_{0}=1$ (see Figure 5.2). This implies that the tumour diffusion rate $\sigma_{1}$ has an important influence on the stability of trivial equilibrium of system (1.3), and system 1.3 can produce spatial patterns for small tumour diffusion rate $\sigma_{1}$.
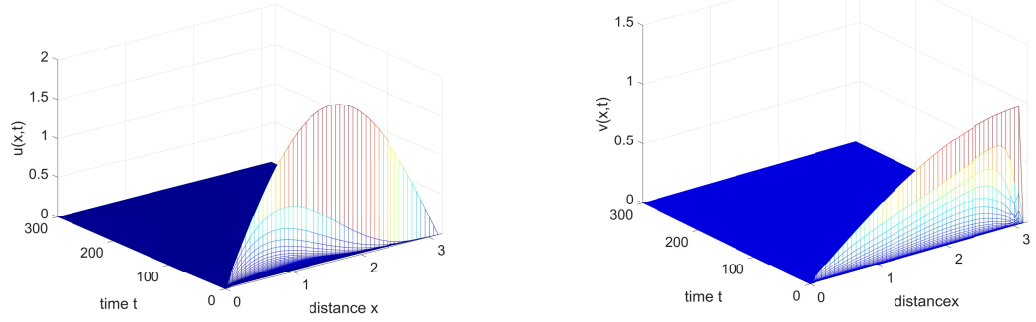

Figure 5.1: Stability of trivial equilibrium $U_{0}=(0,0)$ for system 1.3 with $\sigma_{1}=2$ and initial data $u_{0}=2 \sin x, v_{0}=\sin \frac{x}{2}$.
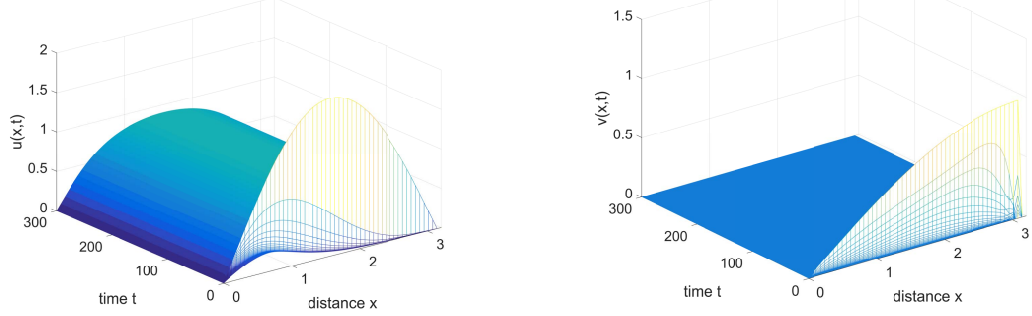

Figure 5.2: Instability of trivial equilibrium $U_{0}=(0,0)$ for system $(1.3)$ with $\sigma_{1}=0.2$ and initial data $u_{0}=2 \sin x, v_{0}=\sin \frac{x}{2}$.

For system (1.4), let $l=1, \sigma_{2}=1.5, p=0.1, s=0.1, q=3.2, e=0.2$. If $\sigma_{1}=0.001,1$, $10^{3}$, then we know from Theorem $2.1(2)$ that the trivial equilibrium $U_{0}=(0,0)$ is always 
unstable, see Figure 5.3. This indicates that the tumour diffusion rate $\sigma_{1}$ cannot affect the instability of trivial equilibrium of system (1.4), that is, system (1.4) can generate spatial patterns for any tumour diffusion rate $\sigma_{1}$.
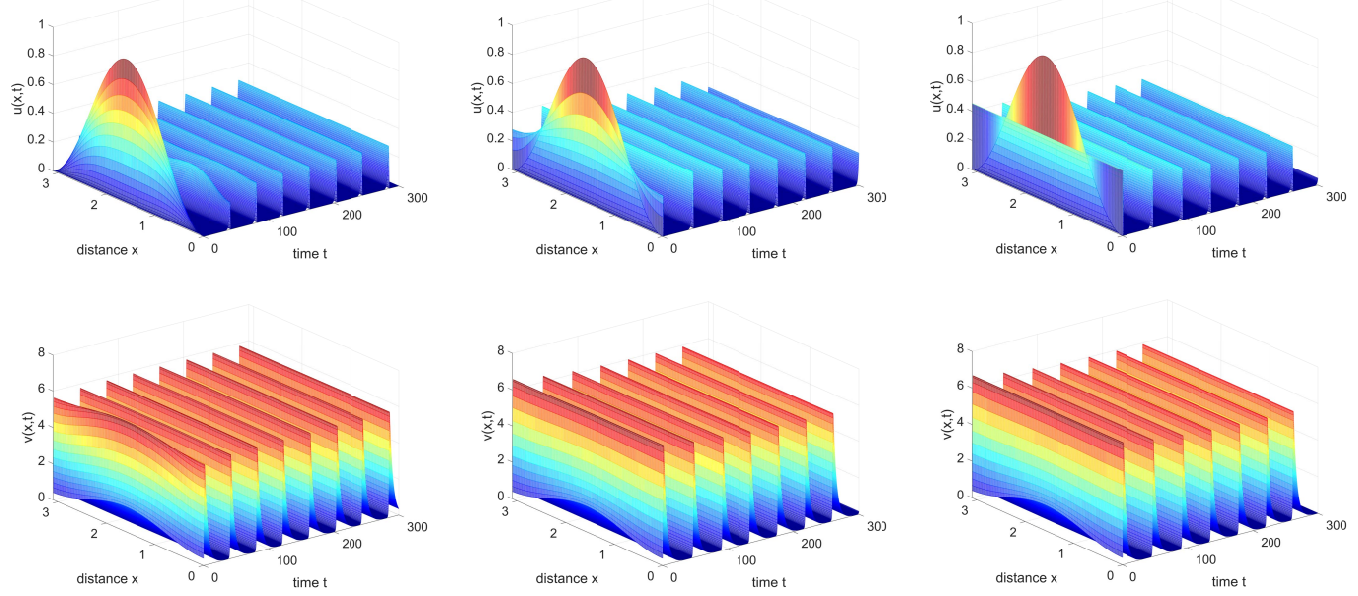

Figure 5.3: Instability of trivial equilibrium $U_{0}=(0,0)$ for system 1.4 with $\sigma_{1}=0.001$, $1,10^{3}$ and initial data $u_{0}=0.5-0.5 \cos (5 x), v_{0}=0.9-0.5 \cos (5 x)$. First column: $\sigma_{1}=0.001$; second column: $\sigma_{1}=1$; third column: $\sigma_{1}=10^{3}$.

Next, we give numerical examples of the existence of steady-state bifurcation solutions of the system (4.1) emitting from $U_{3}^{*}$ in one- and two-dimensional domains, respectively.

\section{(I) One-dimensional simulation.}

(1) By 2.4), we obtain the bifurcation parameter $\delta_{j}^{(3)}=\frac{-u_{3}^{*} l^{2}\left(\sigma_{2} j^{2}+\mathfrak{f}_{3} l^{2}\right)}{\sigma_{2} j^{4}}$, and we plot the curve of $\delta_{j}^{(3)}$ with respect to $j$ in Figure 5.4 . The parameter values are $p=1, s=0.2$, $q=0.59, e=0.1, \sigma_{2}=0.001, l=2$. Moreover, we observe that there are two simple bifurcation points in Figure 5.4 .

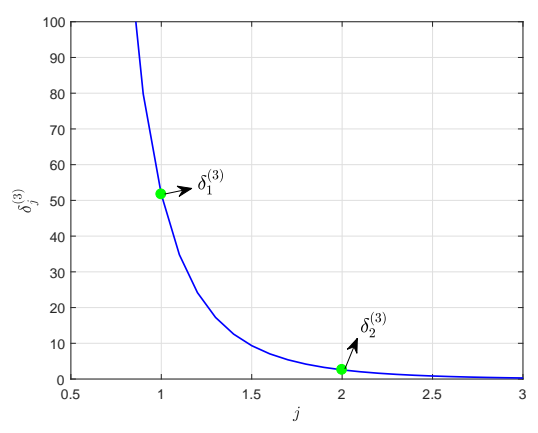

Figure 5.4: Curve of bifurcation parameter for system 4.1. 
(2) Under the parameter values in Figure 5.4, system (4.1) exists positive equilibrium $U_{3}^{*}=\left(u_{3}^{*}, v_{3}^{*}\right)=(0.8851,0.1149)$, and there have two bifurcation points $\left(\delta_{j}^{(3)}, U_{3}^{*}\right), j=1,2$ which are all simple. It follows from Theorem 4.1 that the system (4.1) has at least a positive solution emitting from $U_{3}^{*}$ in the neighborhood of bifurcation points $\left(\delta_{j}^{(3)}, U_{3}^{*}\right)$, $j=1,2$. This means that the effector cells and tumour cells can coexist. Figure 5.5 plots the positive solution of system 4.1 near the bifurcation points $\left(\delta_{j}^{(3)}, U_{3}^{*}\right), j=1,2$, where $\delta_{1}^{(3)}=51.6898, \delta_{2}^{(3)}=2.5668$. In summary, for some diffusion rate $\sigma_{2}$ of effector cells, the variation of diffusion rate $\sigma_{1}$ of tumour cells can destabilize the equilibrium $U_{3}^{*}$ and lead system 4.1 to produce spatial patterns.

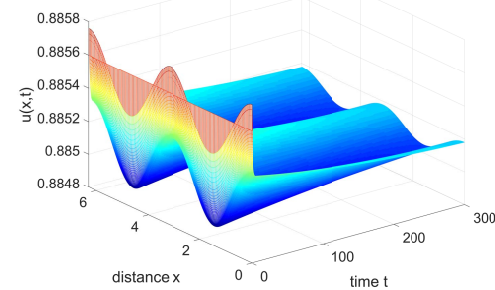

(a)

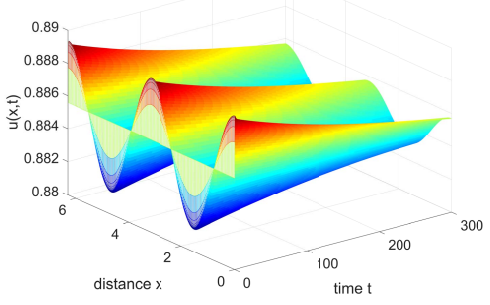

(c)

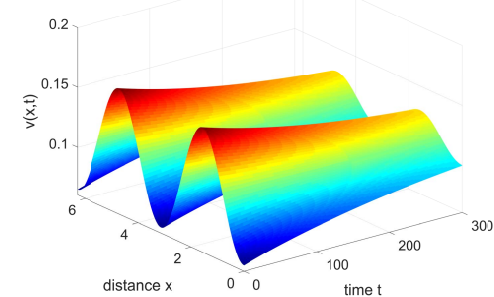

(b)

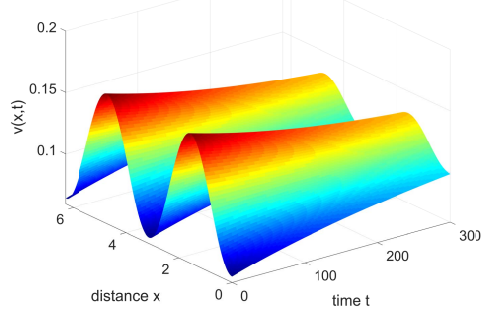

(d)

Figure 5.5: A positive solution for system (4.1) with initial values $u_{0}=0.8851+$ $0.0005 \cos (x), v_{0}=0.1149-0.05 \cos (x)$, where $\sigma_{1}=51.6898$ in $(\mathrm{a})-(\mathrm{b})$ and $\sigma_{1}=2.5668$ in $(\mathrm{c})-(\mathrm{d})$.

On the other hand, from Figure 5.5, we find that the system 4.1) is always in a oscillatory behavior. When $\sigma_{1}$ is small, the oscillation amplitude of tumour cells concentration increases gradually, while the oscillation amplitude of effector cells concentration remains unchanged.

\section{(II) Two-dimensional simulation.}

In this part, we use GMRES algorithm to simulate the spatial pattern of system (4.1) in two-dimensional space domain. In the simulations, the sufficiently small time and space steps are chosen to get the approximate solutions with high precision. In particular, we 
show the different types of diagrams of system (4.1) by taking the different parameter values, initial conditions and spatial domains. By the results of simulations, we observe that the distributions of concentrations of tumour cells and effector cells are always of the same type. Thus, we only restrict our analysis of pattern formation to one distribution (For example, we show the distribution of concentration of the tumour cells $u$ in this paper). Under the parameter values in Figure 5.4, when $\sigma_{1}=51.6898$, the first row of Figure 5.6 presents the distribution of concentration of $u$ in a squared domain $[0,50] \times[0,50]$. When $\sigma_{1}=2.5668$, the second row shows the distribution of concentration of $u$ in a squared domain $[0,6] \times[0,6]$. The initial condition is given by a small perturbation to the value of the positive equilibrium $u_{3}^{*}$, which is more general from the biological point of view. The different colors represent the different values of concentration of $u$ in Figure 5.6. Moreover, we see from Figure 5.6 that the spot-like pattern prevail over the whole domain. This implies that the diffusion rate $\sigma_{1}$ of tumour cells plays an important role in the formation of patterns.
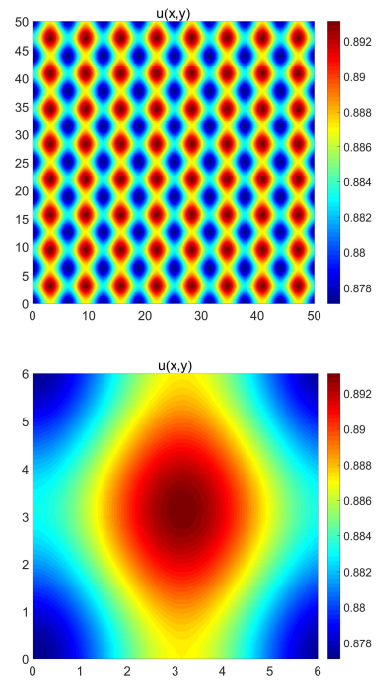
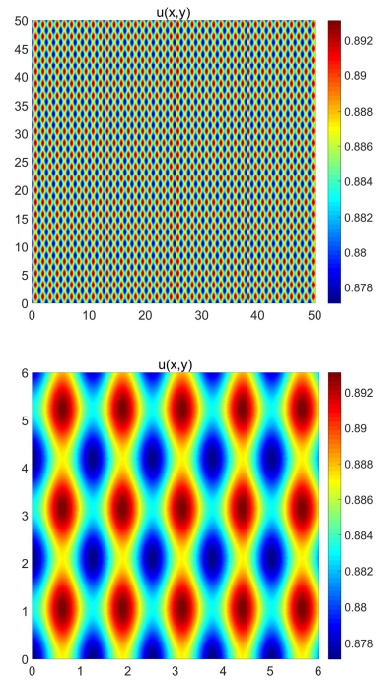
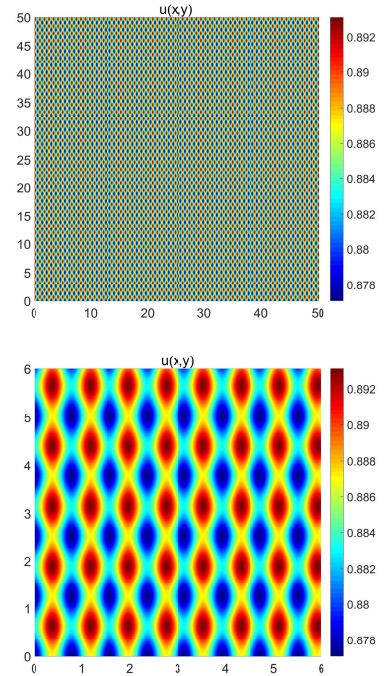

Figure 5.6: Two-dimensional simulations of system 4.1 with $\sigma_{1}=51.6898$ in the first row and $\sigma_{1}=2.5668$ in the second row. The initial data are $u_{0}=0.8851-0.005 \cos (x)-$ $0.003 \cos (y)$ in the first column, $u_{0}=0.8851-0.005 \cos (5 x)-0.003 \cos (3 y)$ in the second column and $u_{0}=0.8851-0.005 \cos (8 x)-0.003 \cos (5 y)$ in the third column.

\section{Conclusion and future work}

In tumour-immune models, pattern formation can be used to judge the proliferation and death of tumour cells over time. Since boundary conditions can affect stability of the trivial equilibrium and thus affect pattern formation of the system, and steady-state bifurcation 
can generate a wealth of spatial patterns, we analyze the effect of boundary conditions on the stability of equilibria, the conditions of steady-state bifurcation occur and spatial patterns produced by bifurcation in this paper. Moreover, we also discuss the long time behavior of solutions to system (1.4). The corresponding results are as follows.

1. Effect of boundary conditions on the stability of equilibria: In Theorem 2.1, we derive that the equilibrium $U_{0}$ for system 1.3 with the homogeneous Dirichlet boundary conditions is stable when $\sigma_{1}>1 / \mu_{0}$ and unstable when $\sigma_{1}<1 / \mu_{0}$, and for system (1.4) with the homogeneous Neumann boundary conditions is always unstable. This implies that the homogeneous Neumann boundary conditions can cause system to produce more spatial patterns than the homogeneous Dirichlet boundary conditions. In fact, combined with homogeneous Neumann boundary conditions and diffusion effects, we obtain that the system can produce the pattern of periodic oscillation in one-dimensional domain (see Figure 5.5) and the spot-like pattern prevail over the whole two-dimensional domain (see Figure 5.6).

2. Steady-state bifurcation and spatial patterns: From Theorems 4.1, 4.4 and 4.5 we find that the tumour diffusion rate $\sigma_{1}$ has an important effect on the existence of the positive solution and spatial patterns for system (4.1). Moreover, we also analyze the stability of bifurcation solution by computing the second derivative $\sigma_{1}^{\prime \prime}(0)$, which is different from the conventional approach that it only needs to consider the first derivative $\sigma_{1}^{\prime}(0)$ (see Theorem 4.6). Biologically, it shows that the effector cells can specifically bind with some tumour cells, so that the tumour cells can be decomposed and cannot reproduce or be exposed. However, tumour cells can not be completely eliminated by the effector cells. From Theorems 4.1, 4.4 4.6 and the spatial patterns exhibited in numerical simulations, we see that the tumour cells will not increase infinitely, the concentrations of tumour cells and effector cells fluctuate periodically in one-dimensional space domain and fluctuate in the shape of spots in two-dimensional space domain. This also implies that the tumour cells and effector cells can coexist and the body can survive.

3. Long time behavior of solutions: Long time behavior of solutions to system (1.4) reflects the survival state of tumour cells and effect cells in the system after a long time, which is an important part of the study of tumour growth. Therefore, we discuss the long time behavior of solutions to system $(1.4)$ in Theorem 3.1. We get that tumour cells will only exist in the body when the steepness $p$ of immune response and the death rate $e$ of effector cells are fixed and the maximum immune response rate $q$ is small. Moreover, we see from Theorem 3.1 that the concentration of tumour cells does not increase indefinitely, but tends to stabilize over time.

When the interactions between tumour cells and immune system are more complex, for example, the other types of cytokines (e.g., interleukin 2, interferon-alpha, etc.) or 
cells (e.g., natural killer cells, cytotoxic $\mathrm{T}$ lymphocytes, $\mathrm{CD}^{+} \mathrm{T}$ cells, etc.) or other environmental factors (e.g., time delay, noise, etc.) are also involved in the immune response, the models (1.1)-1.4 at this time are not enough to meet the current needs. Thus the more practical models must be established, see the modified models of models (1.1) - 1.4) in 24, 28,36. However, some theoretical methods in this paper can be applied to these models to study the interactions between different substances in models. These will be our future works.

\section{References}

[1] J. A. Adam, The dynamics of growth-factor-modified immune response to cancer growth: One-dimensional models, Math. Comput. Modelling 17 (1993), no. 3, 83106.

[2] R. F. Alvarez, J. A. M. Barbuto and R. Venegeroles, A nonlinear mathematical model of cell-mediated immune response for tumor phenotypic heterogeneity, J. Theoret. Biol. 471 (2019), 42-50.

[3] F. Ansarizadeh, M. Singh and D. Richards, Modelling of tumor cells regression in response to chemotherapeutic treatment, Appl. Math. Model. 48 (2017), 96-112.

[4] P. Bi and S. Ruan, Bifurcations in delay differential equations and applications to tumor and immune system interaction models, SIAM J. Appl. Dyn. Syst. 12 (2013), no. $4,1847-1888$.

[5] J. Blat and K. J. Brown, Global bifurcation of positive solutions in some systems of elliptic equations, SIAM J. Math. Anal. 17 (1986), no. 6, 1339-1353.

[6] M. G. Crandall and P. H. Rabinowitz, Bifurcation from simple eigenvalues, J. Functional Analysis 8 (1971), 321-340.

[7] S. Cui and J. Zheng, On eigenvalues of the linearization of a free boundary problem modeling two-phase tumor growth, J. Math. Anal. Appl. 470 (2019), no. 2, 954-981.

[8] I. Elaff, Comparative study between spatio-temporal models for brain tumor growth, Biochem. Biophys. Res. Commun. 496 (2018), no. 4, 1263-1268.

[9] A. Elazab, A. M. Anter, H. Bai, Q. Hu, Z. Hussain, D. Ni, T. Wang and B. Lei, An optimized generic cerebral tumor growth modeling framework by coupling biomechanical and diffusive models with treatment effects, Appl. Soft Comput. 80 (2019), 617-627. 
[10] A. Friedman and K.-Y. Lam, Analysis of a free-boundary tumor model with angiogenesis, J. Differential Equations 259 (2015), no. 12, 7636-7661.

[11] H. Garcke, K. F. Lam and E. Rocca, Optimal control of treatment time in a diffuse interface model of tumor growth, Appl. Math. Optim. 78 (2018), no. 3, 495-544.

[12] D. Gilbarg and N. S. Trudinger, Elliptic Partial Differential Equations of Second Order, Classics in Mathematics, Springer-Verlag, Berlin, 2001.

[13] H. P. Greenspan, Models for the growth of a solid tumor by diffusion, Stud. Appl. Math. 51 (1972), no. 4, 317-340.

[14] G. Guo, B. Li, M. Wei and J. Wu, Hopf bifurcation and steady-state bifurcation for an autocatalysis reaction-diffusion model, J. Math. Anal. Appl. 391 (2012), no. 1, $265-277$.

[15] J. Jang, W.-M. Ni and M. Tang, Global bifurcation and structure of Turing patterns in the 1-D Lengyel-Epstein model, J. Dynam. Differential Equations 16 (2004), no. 2, $297-320$.

[16] Y. Jia, Computational analysis on Hopf bifurcation and stability for a consumerresource model with nonlinear functional response, Nonlinear Dynam. 94 (2018), no. 1, 185-195.

[17] Y. Jia, Y. Li and J. Wu, Qualitative analysis on positive steady-states for an autocatalytic reaction model in thermodynamics, Discrete Contin. Dyn. Syst. 37 (2017), no. $9,4785-4813$.

[18] J. Jiang, H. Wu and S. Zheng, Well-posedness and long-time behavior of a nonautonomous Cahn-Hilliard-Darcy system with mass source modeling tumor growth, J. Differential Equations 259 (2015), no. 7, 3032-3077.

[19] W. Jiang, H. Wang and X. Cao, Turing instability and Turing-Hopf bifurcation in diffusive Schnakenberg systems with gene expression time delay, J. Dynam. Differential Equations 31 (2019), no. 4, 2223-2247.

[20] V. Kuznetsov, I. Makalkin, M. Taylor and A. Perelson, Nonlinear dynamics of immunogenic tumours: Parameter estimation and global bifurcation analysis, Bull. Math. Biol. 56 (1994), no. 2, 295-321.

[21] S. Li, J. Wu and H. Nie, Steady-state bifurcation and Hopf bifurcation for a diffusive Leslie-Gower predator-prey model, Comput. Math. Appl. 70 (2015), no. 12, 30433056 . 
[22] B. Niu, Y. Guo and Y. Du, Hopf bifurcation induced by delay effect in a diffusive tumor-immune system, Internat. J. Bifur. Chaos Appl. Sci. Engrg. 28 (2018), no. 11, 1850136, 14 pp.

[23] R. A. Ramos, J. Zapata, C. A. Condat and T. S. Deisboeck, Modeling cancer immunotherapy: Assessing the effects of lymphocytes on cancer cell growth and motility, Phys. A 392 (2013), no. 10, 2415-2425.

[24] F. A. Rihan, D. H. Abdel Rahman, S. Lakshmanan and A. S. Alkhajeh, A time delay model of tumour-immune system interactions: Global dynamics, parameter estimation, sensitivity analysis, Appl. Math. Comput. 232 (2014), 606-623.

[25] R. D. Schreiber, L. J. Old and M. J. Smyth, Cancer immunoediting: Integrating immunity's roles in cancer suppression and promotion, Science 331 (2011), no. 6024, $1565-1570$.

[26] J. Shi and X. Wang, On global bifurcation for quasilinear elliptic systems on bounded domains, J. Differential Equations 246 (2009), no. 7, 2788-2812.

[27] J. Smoller, Shock Waves and Reaction-Diffusion Equations, Second edition, Grundlehren der Mathematischen Wissenschaften 258, Springer-Verlag, New York, 1994.

[28] K. E. Starkov and A. P. Krishchenko, On the global dynamics of one cancer tumour growth model, Commun. Nonlinear Sci. Numer. Simul. 19 (2014), no. 5, 1486-1495.

[29] B. Su, W. Zhou, K. S. Dorman and D. E. Jones, Mathematical modelling of immune response in tissues, Comput. Math. Methods Med. 10 (2009), no. 1, 9-38.

[30] W. Suo and Y. Jia, Effects of the killing rate on global bifurcation in an oncolytic-virus system with tumors, J. Appl. Anal. Comput. 7 (2017), no. 1, 264-277.

[31] J. Wang and Y. Jia, Analysis on bifurcation and stability of a generalized Gray-Scott chemical reaction model, Phys. A 528 (2019), 121394, 11 pp.

[32] J. Wang, H. Zheng and Y. Jia, Existence and bifurcation of non-constant positive steady states for a tumor-immune model, Z. Angew. Math. Phys. Accepted, 2020.

[33] Y. Wang, J. Wu and Y. Jia, Steady-state bifurcation for a biological depletion model, Internat. J. Bifur. Chaos Appl. Sci. Engrg. 26 (2016), no. 4, 1650066, 15 pp.

[34] D. Wodarz and N. Komarova, Computational Biology of Cancer: Lecture Notes and Mathematical Modelling, World Scientific, Singapore, 2005. 
[35] J. Wu, Stationary solutions of a free boundary problem modeling the growth of tumors with Gibbs-Thomson relation, J. Differential Equations 260 (2016), no. 7, 5875-5893.

[36] J. Yang, Y. Tan and R. A. Cheke, Thresholds for extinction and proliferation in a stochastic tumour-immune model with pulsed comprehensive therapy, Commun. Nonlinear Sci. Numer. Simul. 73 (2019), 363-378.

Jingjing Wang and Hongchan Zheng

School of Mathematics and Statistics, Northwestern Polytechnical University, Xi'an, Shaanxi 710072, China

E-mail addresses: jjwang@mail.nwpu.edu.cn, zhenghc@nwpu.edu.cn

Yunfeng Jia

School of Mathematics and Information Science, Shaanxi Normal University, Xi'an, Shaanxi 710062, China

E-mail address: jiayf@snnu.edu.cn

Hong-Kun Xu

School of Science, Hangzhou Dianzi University, Hangzhou, Zhejiang 310018, China E-mail address: xuhk@hdu.edu.cn 